\title{
The length scale equation in turbulence models
}

\author{
L. H. Kantha \\ Department of Aerospace Engineering Sciences and Program in Atmospheric and Oceanic Sciences, University of Colorado, \\ Boulder, CO 80309-0431, USA
}

Received: 14 January 2003 - Revised: 2 April 2003 - Accepted: 3 April 2003 - Published: 25 February 2004

Part of Special Issue "Dedicated to Prof. A. D. Kirwan Jr. on the occasion of his 70th birthday"

\begin{abstract}
The question of which length scale equation to use in models of turbulence has long been controversial and several approaches have been suggested and used in the past. In this paper, we demonstrate that all these approaches are equivalent and the inconsistencies in the use of some of these approaches arise from improper modeling of the diffusion term in the length scale equation. We formulate a general length scale equation, which encompasses all current approaches. This equation is devoid of inconsistencies and should prove useful in its general form, or one of its subsets, in turbulence closure modeling.
\end{abstract}

\section{Introduction}

The weakest link in second moment turbulence closure has always been the specification of the turbulence macroscale $\ell$. If one accepts the second moment closure methodology as one of the currently available methods of some practical utility, if not conceptual elegance or physical unassailabilty, then it is natural to ask what its strengths and weaknesses are, and how one can remedy the latter. In this sense, the question of the turbulence length scale and how to model it properly are still outstanding issues in second moment closure. Here, we look at the many approaches that have been proposed and ascertain why certain approaches are problematic. We derive an equation for a generalized quantity involving the length scale that in turn encompasses all the current approaches properly, and hence can be used for whatever approach one chooses.

The bare minimum description of turbulence requires two quantities: 1 . The intensity of turbulence indicated by the kinetic energy in the turbulent fluctuations, or equivalently, the turbulence velocity scale $q$, and 2 . The scale around which this energy is concentrated, represented by the peak in the turbulence spectrum, or equivalently the turbulence length scale $\ell$. It was Ludwig Prandtl who pioneered the use of one-equation turbulence models in which an equation for the turbulence kinetic energy (TKE) is solved but the

Correspondence to: L. H. Kantha

(kantha@colorado.edu) length scale is suitably prescribed (Prandtl, 1945). However, it was Kolmogoroff, who was the first to propose the use of two-equation models in which equations for both the TKE and a quantity involving the length scale are solved (Kolmogoroff, 1942). Since then, considerable progress has been made in modeling turbulence, culminating in the currently popular second moment closure models involving differential equations for TKE and a quantity involving $\ell$, along with algebraic expressions for the second moment quantities, derived by appropriate simplification of the full set of 10 partial differential equations for the second moment turbulence quantities Reynolds stress $\tau_{i j}$, turbulent heat flux $q_{i}$ and the temperature variance $\overline{\theta^{2}}$. These models are called algebraic stress models (Level 2.5 in Mellor and Yamada 1974 terminology). We will not discuss algebraic stress models here, but instead concentrate on the length scale equation. Interested readers are referred to Cheng et al. (2002), Kantha and Clayson (1994), Galperin et al. (1988), Rodi (1987), Mellor and Yamada (1974, 1982), and Hanjalic and Launder (1974). Mellor and Herring (1973), a dated but still insightful survey of turbulence closure, touches upon some of the issues discussed in this paper. The reader is also referred to Kantha (2003) for the latest update of the second moment closure constants that removes some of the problems that were evident in the original Mellor and Yamada (1982) closure. In particular, a better agreement with the observed wind velocity profile in the atmospheric surface layer is obtained from the use of the updated constants.

There is no ambiguity in the proper form of the TKE equation (Mellor and Yamada, 1982; Galperin et al., 1988; Kantha and Clayson, 1994; Baumert and Peters, 2000), which can be readily derived from the equations for the Reynolds stresses. We write down The TKE equation here for future reference:

$$
\begin{aligned}
\frac{\mathrm{D} k}{\mathrm{D} t}-\frac{\partial}{\partial x_{j}}\left[\frac{v_{t}}{\sigma_{k}} \frac{\partial k}{\partial x_{j}}\right] & =P+B-\varepsilon \\
& =-\overline{u_{j} u_{k}} \frac{\partial U_{j}}{\partial x_{k}}-\beta g_{j} \overline{u_{j} \theta}-\varepsilon
\end{aligned}
$$


where $P$ is the shear production, $B$ is the buoyancy production and $\varepsilon$ is the dissipation rate of $\operatorname{TKE}(k)$. The quantity $v_{t}=C_{\mu} k^{2} / \varepsilon$ is the turbulent viscosity and $\sigma_{k}$ is the TKE Prandtl number. $\mathrm{D} / \mathrm{D} t$ is the substantial derivative and the second term on the left hand side is the turbulent diffusion term, modeled as down-the-gradient transport of TKE. The traditional value for $\sigma_{k}$ is 1.0 . Almost all $k-\varepsilon$ modelers have used $C_{\mu}=0.09$.

For thin horizontal shear layers, this equation becomes:

$$
\begin{aligned}
\frac{\mathrm{D} k}{\mathrm{D} t}-\frac{\partial}{\partial z}\left[\frac{v_{t}}{\sigma_{k}} \frac{\partial k}{\partial z}\right] & =P+B-\varepsilon \\
& =-\overline{u w} \frac{\partial U}{\partial z}+\beta g \overline{w \theta}-\varepsilon
\end{aligned}
$$

where $\partial U / \partial z$ is the shear $(z$ is in the vertical direction and perpendicular to the shear flow). This can also be written as:

$$
\begin{array}{r}
\frac{\mathrm{D}}{\mathrm{D} t}\left(q^{2}\right)-\frac{\partial}{\partial z}\left[q l S_{q} \frac{\partial}{\partial z}\left(q^{2}\right)\right]=2(P+B-\varepsilon) \\
=-2 \overline{u w} \frac{\partial U}{\partial z}+2 \beta g \overline{w \theta}-2 \frac{q^{3}}{B_{1} l}
\end{array}
$$

where we have put $\varepsilon=q^{3} /\left(B_{1} \ell\right)$ making use of the fact that in a turbulent flow, the dissipation rate is set by the properties of the energy containing eddies and is therefore proportional to $q^{3} / \ell$ (Kolmogoroff 1942). $B_{1}$ is a closure constant with a traditional value of 16.6 (Mellor and Yamada, 1982; Kantha and Clayson, 1994) and is related to $C_{\mu}$ by

$C_{\mu}=4 B_{1}^{-4 / 3} ; \quad S_{q}=\frac{B_{1} C_{\mu}}{4 \sigma_{k}}=\frac{1}{\sigma_{k} B_{1}^{1 / 3}}$.

The only controversy surrounding the TKE equation is the manner in which the diffusion term is modeled. It has long been known that down-the-gradient approximation for turbulent diffusion is not always applicable, and this approximation has been severely criticized by turbulence purists (for example, Corrsin, 1979), since large scale eddies are indeed capable of transporting properties against the gradient (Townsend, 1980). Still the practice persists, and is often justified by the fact that the diffusion terms are generally less important compared to the production and dissipation terms in the equations for the Reynolds stresses. This is the primary reason that the results of second moment closure are relatively insensitive to the exact model for turbulent diffusion of TKE or the exact value for the diffusion coefficient. This is also why it is possible to obtain reasonable results even though diffusion terms are modeled as downthe-gradient transport terms, as almost all second moment closure models do. In fact, in many cases, local equilibrium prevails, and the turbulence production is balanced almost exactly by dissipation, and diffusion is nearly zero.

However, the same is not true for the length scale equation. The diffusion terms are not negligible except in the special case of spatially homogeneous turbulence. Nevertheless, tradition prevails, and the diffusion terms in all length scale equations to-date have been modeled as down-the-gradient transport terms. We follow the same practice here, since our objective is to show how the various length scale models can be reconciled within the current framework of second moment closure models.

\section{The length scale models}

There are many ways to prescribe the turbulence macroscale $\ell$. The simplest and the most arbitrary method has been to simply prescribe it arbitrarily by an algebraic equation or some other means. In doing so, one makes use of the well-known and the widely accepted fact that the turbulence macroscale in a boundary layer on a solid surface is simply proportional to the distance from the surface (some believe that this proportionality extends to the viscous sublayer on a smooth wall). Use is also made of the fact that as the edge of the boundary layer is reached, the eddy size tends to be proportional to and a fraction of the thickness of the boundary layer. Thus:

$\ell(z)=\ell_{0}\left(\frac{\kappa z}{\kappa z+\ell_{0}}\right)$

where $\kappa$ is the von Karman constant (equal to 0.4 ), $z$ is the distance from the surface and $\ell_{0}$ is the asymptotic value of $\ell$ towards the edge of the boundary layer. This expression was first proposed by Blackadar (1962), but in the context of a mixing length approach. The asymptotic value is either arbitrarily prescribed as some fraction of the turbulent boundary layer thickness or calculated by the use of a formula such as (Mellor and Durbin, 1975; Martin, 1985, 1986):

$\ell_{0}=\frac{\alpha \int_{0}^{\infty} q z d z}{\int_{0}^{\infty} q d z}$

where $\alpha$ is an empirical constant. In many cases, the results do not appear to be overly sensitive to moderate changes in its value, although there appears to be a fundamental difference (Galperin and Hassid, 1986) between the values that are suitable for non-rotating boundary layers frequently encountered in the laboratory $(\sim 0.55)$ and those for rotating ones characteristic of geophysical flows $(\sim 0.2)$.

While this prescription works well, especially in regions adjacent to a solid surface, no doubt due to the robustness of the assumption near the wall, the method is highly empirical, requires a priori knowledge of the length scale behavior in that particular flow situation. While it is still possible to derive an equivalent length scale in complex flow situations by empirical means, where such empirical data are lacking or are untenable, it is of little practical use. Also in a planetary boundary layer capped by an inversion, contrary to the Blackadar formulation, the length scale variation in the boundary layer must be non-monotonic as the inversion is approached (Cheng and Canuto, 1994). 
It is therefore desirable to write a transport equation for the turbulence length scale or equivalently, a quantity involving it, which is devoid of such arbitrariness and therefore of universal applicability. The various quantities involving the length scale that have physical interpretations are: $q^{3} / \ell$, $q / \ell, \ell / q, q \ell$, and $q^{2} \ell$. The first is proportional to the dissipation rate of TKE, $\varepsilon$. The second and third can be interpreted as being proportional to the turbulence frequency $\omega$ (equivalently $\varepsilon / k$ ) and the turbulence time scale $\tau$ (equivalently $k / \varepsilon$ ), respectively. The fourth one is proportional to the turbulent viscosity $v_{T}$ (equivalently $k^{2} / \varepsilon$ ). The last one represents a spherical average of two-point turbulent correlations integrated over the separation distance (see Sect. 4) and does not have as clear a physical meaning. It appears that Kolmogoroff (1942) was the first to suggest the use of a length scale equation (for turbulence frequency) and since then many such equations have been proposed. However most approaches have had one problem or another, and despite claims to the contrary by various proponents, no one approach is superior to the others, and it is likely that all of these approaches are equivalent.

It is in fact possible to write down a transport equation for the general quantity $q^{m} \ell^{n}$ where $m$ and $n$ are integer values. The various length scale equations then become subsets. A major problem persists however: unlike the TKE equation, all the turbulent correlation terms that appear in any length scale equation must be modeled, that too in the absence of empirical wisdom about the nature or behavior of these quantities. The most favored approach has been to simply mimic the TKE equation so that no matter which quantity is modeled, the equation consists of a shear production term, a buoyancy production term, a dissipation term and a diffusion term, whose forms are similar to those of the corresponding terms in the TKE equation and related to them by simple dimensional considerations. Here we will develop a transport equation for $q^{m} \ell^{n}$ and using its subsets, demonstrate that it is the way the diffusional term is modeled that introduces well-known difficulties in some of the traditional approaches. But first we will describe how the different equations are formulated so as to have a better understanding of the inter-relationships between the various approaches.

\section{The $\varepsilon\left(\right.$ or $\left.\mathbf{q}^{3} / \mathbf{l}\right)$ equation}

An exact equation for the isotropic dissipation rate of TKE can be derived by differentiating the Navier-Stokes equation for the turbulent velocity component $u_{i}$ w.r.t. $x_{k}$, multiplying by $v \partial u_{i} / \partial x_{k}$ and ensemble averaging to obtain a transport equation for the isotropic dissipation rate $\varepsilon_{I}=\overline{v\left(\partial u_{i} / \partial x_{k}\right)^{2}}$ (Harlow and Nakayama, 1967; Hanjalic and Launder, 1972):

$$
\begin{array}{r}
\frac{\mathrm{D} \varepsilon_{I}}{\mathrm{D} t}=-2 v \frac{\partial U_{j}}{\partial x_{k}}\left(\overline{\frac{\partial u_{j}}{\partial x_{l}} \frac{\partial u_{k}}{\partial x_{l}}+\frac{\partial u_{l}}{\partial x_{j}} \frac{\partial u_{l}}{\partial x_{k}}}\right)-2 v \overline{\frac{\partial u_{j}}{\partial x_{k}} \frac{\partial u_{j}}{\partial x_{l}} \frac{\partial u_{k}}{\partial x_{l}}} \\
-v \frac{\partial}{\partial x_{k}}\left(\overline{u_{k} \frac{\partial u_{j}}{\partial x_{k}} \frac{\partial u_{j}}{\partial x_{k}}}\right)-v \frac{\partial}{\partial x_{j}}\left(\overline{\frac{1}{\rho} \frac{\partial p}{\partial x_{l}} \frac{\partial u_{j}}{\partial x_{l}}}\right)
\end{array}
$$

The next step is to invoke asymptoticity at high turbulence Reynolds numbers and equate $\varepsilon_{I}$ to the dissipation rate $\varepsilon=q^{3} /\left(B_{1} \ell\right)$. Thus the equation, though derived for a process representative of the small universal Kolmogoroff viscous scales of turbulence, involves a quantity that is nevertheless a function solely of the large energy containing scales and is therefore a meaningful and legitimate equation for the turbulence macroscale $\ell$. This is because of the remarkable property of turbulence that the dissipation rate of TKE is not a function, asymptotically, of the Kolmogoroff viscous dissipation scales, which merely adjust to accommodate the energy cascade from large scales to small scales across the wavenumber spectrum.

This equation is in more extensive use today than any other length scale equation. Its proponents claim that it is more meaningful and useful than others because it pertains to a physically meaningful and rather fundamental property of turbulence, namely the dissipation rate $\varepsilon$. On the other hand, Mellor (1985) shows that Eq. (7) can also be derived from an equation for two-point correlation of the turbulence velocity component by taking the limit of zero separation distance. He argues that therefore the equation is really an equa- tion for dissipation scales of turbulence and not a macroscale, and besides contains little new information than the original two-point correlation equation from which it can be derived. Interpreted this way, there would appear to be a major flaw in this approach. But if we invoke the fact that the dissipation rate is really a function of large scales, then Eq. (7) is a pseudo equation for $q^{3} / \ell$ and in this sense an acceptable equation for $\ell$.

The next task is to model the terms on the right hand side of Eq. (7). Unfortunately, all the terms have to be modeled and herein lies the problem. The first term can be identified as the production term, and the last two terms as diffusional terms, while the third term is the dissipation rate (of the dissipation rate!). The second term is interpreted as the generation rate of vorticity fluctuations and modeled together with the dissipation term (Hanjalic and Launder, 1972). Without going into details (but see, Hanjalic and Launder, 1972, for example), the modeled equation can be written as:

$$
\begin{aligned}
\frac{\mathrm{D} \varepsilon}{\mathrm{D} t}- & \frac{\partial}{\partial x_{k}}\left[C_{\mu} \frac{k}{\varepsilon}\left(-\overline{u_{k} u_{l}} \frac{\partial \varepsilon}{\partial x_{l}}\right)\right]=P_{\varepsilon}+B_{\varepsilon}-\varepsilon_{\varepsilon} \\
& =\frac{\varepsilon}{k}\left[C_{\varepsilon 1}\left(-\overline{u_{j} u_{k}} \frac{\partial U_{j}}{\partial x_{k}}\right)+C_{\varepsilon 3}(\beta g \overline{w \theta})-C_{\varepsilon 2} \varepsilon\right]
\end{aligned}
$$


where $P_{\varepsilon}, B_{\varepsilon}$ and $\varepsilon_{\varepsilon}$ are the shear production, the buoyancy production and the dissipation of the dissipation rate $\varepsilon$, respectively. For thin horizontal shear layers, this equation reduces to:

$$
\begin{aligned}
\frac{\mathrm{D} \varepsilon}{\mathrm{D} t}- & \frac{\partial}{\partial z}\left[\frac{v_{t}}{\sigma_{\varepsilon}} \frac{\partial \varepsilon}{\partial z}\right]=P_{\varepsilon}+B_{\varepsilon}-\varepsilon_{\varepsilon} \\
& =\frac{\varepsilon}{k}\left[C_{\varepsilon 1}\left(-\overline{u w} \frac{\partial U}{\partial z}\right)+C_{\varepsilon 3}(\beta g \overline{w \theta})-C_{\varepsilon 2} \varepsilon\right]
\end{aligned}
$$

where $\partial U / \partial z$ is the shear as before in Eq. (2), and $\sigma_{\varepsilon}$ is the dissipation Prandtl number.

For future reference, we will also write this equation in terms of $q$ and $\ell$ by putting $\varepsilon=q^{3} /\left(B_{1} \ell\right)$ :

$$
\begin{aligned}
& \frac{\mathrm{D}}{\mathrm{D} t}\left(\frac{q^{3}}{\ell}\right)-\frac{\partial}{\partial z}\left[q \ell S_{\ell \varepsilon} \frac{\partial}{\partial z}\left(\frac{q^{3}}{\ell}\right)\right] \\
& \quad=P_{q^{3} / \ell}+B_{q^{3} / \ell}-\varepsilon_{q^{3} / \ell} \\
& \quad=\frac{q}{\ell}\left[E_{\varepsilon 1}\left(-\overline{u w} \frac{\partial U}{\partial z}\right)+E_{\varepsilon 3}(\beta g \overline{w \theta})-E_{\varepsilon 2} \frac{q^{3}}{B_{1} \ell}\right]
\end{aligned}
$$

The constants $C_{\varepsilon 1}, C_{\varepsilon 2}, C_{\varepsilon 3}$ and $\sigma_{\varepsilon}$ and $E_{\varepsilon 1}, E_{\varepsilon 2}, E_{\varepsilon 3}$, and $S_{\ell \varepsilon}$ are related:

$$
\begin{aligned}
E_{\varepsilon 1} & =2 C_{\varepsilon 1}, \quad E_{\varepsilon 2}=2 C_{\varepsilon 2}, \quad E_{\varepsilon 3}=2 C_{\varepsilon 3} \\
S_{\ell \varepsilon} & =\frac{B_{1} C_{\mu}}{4 \sigma_{\varepsilon}}=\frac{1}{\sigma_{\varepsilon} B_{1}^{1 / 3}}
\end{aligned}
$$

While a range of values have been used for the closure constants (Rodi, 1987; Tennekes, 1989; Speziale et al., 1990; Burchard and Bolding, 2001), the traditional values for these constants are:

$C_{\varepsilon 1}=1.46, \quad C_{\varepsilon 2}=1.92, \quad C_{\varepsilon 3}=0.2$ and $\sigma_{\varepsilon}=1.159$

Of these, there is the least general agreement on the value of $\sigma_{\varepsilon}$, with the values ranging from 1.11 (Rodi, 1987) to 0.8 (Zeierman and Wolfshtein, 1986) and that of $C_{\varepsilon 3}$ whose value ranges from -1.4 to +1.45 (see Table 4 in Baumert and Peters, 2000). However, theoretical considerations, as pointed out below suggest that:

$C_{\varepsilon 1}=3 / 2, \quad C_{\varepsilon 2}=2$,

Therefore,

$E_{\varepsilon 1}=3, \quad E_{\varepsilon 2}=4$,

Speziale et al. (1990) point out a major problem associated with the $k-\varepsilon$ two-equation turbulence model. It is associated with the fact that a lack of natural boundary conditions for the dissipation rate $\varepsilon$ makes it difficult to derive and apply a boundary condition that is consistent asymptotically or is not numerically stiff to integrate the equations all the way to a solid boundary. Accurate and robust low Reynolds number near-wall corrections needed to integrate down to the wall have therefore been hard to derive. But where integration to the boundary is not needed, the model is well behaved and in extensive use.

\section{The $q^{2} l$ equation}

This the next most popular approach, used extensively in geophysical applications. It is also one of the most controversial.

Rotta (1951, see also 1972) appealed to the two-point correlation equations that can be derived from the Navier-Stokes equation by multiplying the equation for $u_{i}(\boldsymbol{x})$ by $u_{i}(\boldsymbol{x}+\boldsymbol{r})$ and adding to the equation for $u_{i}(\boldsymbol{x}+\boldsymbol{r})$ multiplied by $u_{i}(\boldsymbol{x})$ and taking an ensemble average. The quantity $q^{2} \ell$ can then be defined as a volume integral of the two-point correlation:

$q^{2} \ell=\alpha \iiint_{V} \overline{u_{j}(\boldsymbol{x}) u_{j}(\boldsymbol{x}+\boldsymbol{r})} \frac{d v}{r^{2}}$

and the corresponding equation for $q^{2} \ell$ is (Wolfstein et al., 1974; Naot et al., 1973; see also Lewellen, 1977):

$$
\begin{aligned}
& \frac{\mathrm{D}}{\mathrm{D} t}\left(q^{2} \ell\right) \sim \\
& \iiint_{V}\left\{\left[U_{k}(\boldsymbol{x})-U_{k}(\boldsymbol{x}+\boldsymbol{r})\right] \frac{\partial}{\partial r_{k}}\left(\overline{u_{j}(\boldsymbol{x}) u_{j}(\boldsymbol{x}+\boldsymbol{r})}\right)-\overline{u_{j}(\boldsymbol{x}) u_{k}(\boldsymbol{x}+\boldsymbol{r})} \frac{\partial}{\partial x_{k}} U_{j}(\boldsymbol{x}+\boldsymbol{r})+\overline{u_{j}(\boldsymbol{x}) u_{k}(\boldsymbol{x}+\boldsymbol{r})} \frac{\partial}{\partial x_{k}} U_{j}(\boldsymbol{x})\right. \\
& \quad-\frac{1}{\alpha}\left(\frac{\partial}{\partial x_{k}} \overline{u_{k}(\boldsymbol{x}) u_{j}(\boldsymbol{x}) u_{j}(\boldsymbol{x}+\boldsymbol{r})}+\frac{\partial}{\partial r_{k}} \overline{u_{j}(\boldsymbol{x}) u_{k}(\boldsymbol{x}+\boldsymbol{r}) u_{j}(\boldsymbol{x}+\boldsymbol{r})}+\frac{\partial}{\partial r_{k}} \overline{u_{j}(\boldsymbol{x}) u_{k}(\boldsymbol{x}) u_{j}(\boldsymbol{x}+\boldsymbol{r})}\right) \\
& \quad-\frac{1}{\alpha \rho}\left(\frac{\partial}{\partial x_{j}} \overline{p(\boldsymbol{x}) u_{j}(\boldsymbol{x}+\boldsymbol{r})}+\frac{\partial}{\partial r_{j}} \overline{p(\boldsymbol{x}+\boldsymbol{r}) u_{j}(\boldsymbol{x})}-\frac{\partial}{\partial r_{j}} \overline{p(\boldsymbol{x}) u_{j}(\boldsymbol{x}+\boldsymbol{r})}\right) \\
& \left.\quad-v \frac{\partial^{2}}{\partial x_{k} \partial x_{k}} \overline{u_{j}(\boldsymbol{x}) u_{j}(\boldsymbol{x}+\boldsymbol{r})}-2 v \frac{\partial^{2}}{\partial x_{k} \partial r_{k}} \overline{u_{j}(\boldsymbol{x}) u_{j}(\boldsymbol{x}+\boldsymbol{r})}+2 v \frac{\partial^{2}}{\partial r_{k} \partial r_{k}} \overline{u_{j}(\boldsymbol{x}) u_{j}(\boldsymbol{x}+\boldsymbol{r})}\right\} \frac{d v}{r^{2}}
\end{aligned}
$$

without the body force terms. It is readily apparent that all the terms on the right hand side must be modeled. The physical interpretation of various terms is difficult at best, although viscous and diffusion terms can be identified. Nevertheless, the various terms can be modeled in the traditional manner to yield for a horizontal thin shear layer, after the inclusion 
of the buoyancy term in the derivation (Mellor and Yamada, 1982):

$$
\begin{aligned}
\frac{\mathrm{D}}{\mathrm{D} t}\left(q^{2} \ell\right)-\frac{\partial}{\partial z}\left[q \ell S_{\ell L} \frac{\partial}{\partial z}\left(q^{2} \ell\right)\right] & =P_{q^{2} \ell}+B_{q^{2} \ell}-\varepsilon_{q^{2} \ell} \\
& =\ell\left\{E_{L 1}\left(-\overline{u w} \frac{\partial U}{\partial z}\right)+E_{L 3}(\beta g \overline{w \theta})-E_{L 2} \frac{q^{2}}{B_{1} \ell}\left[1+E_{L 4}\left(\frac{\ell}{\kappa L}\right)^{2}\right]\right\}
\end{aligned}
$$

where the dissipation term contains an additional near-wall correction term that has been tacked on; $L$ is the distance from a solid surface. This wall-term is absolutely essential to force the model to be consistent with the length scale variation in the constant flux region adjacent to a boundary in a neutrally stratified flow. This has been cited as a major flaw in this approach (Rodi, 1987). Nevertheless, this equation has been used extensively in geophysical applications (Mellor and Yamada, 1982; Kantha and Clayson, 1994; Rosati and Miyakoda, 1988). Note that such a correction term was not necessary in the $\varepsilon$ equation.

The traditional values for the constants in this equation are:

$E_{L 1}=1.8, \quad E_{L 2}=1.0, \quad E_{L 3}=1.8$,

$E_{L 4}=1.33, \quad S_{\ell L}=0.2$

where the non-zero value of $E_{L 4}$ is forced upon by the constraint on the length scale behavior in the constant flux region:

$E_{L 1}=E_{L 2}\left(1+E_{L 4}\right)-\kappa^{2} B_{1} S_{\ell L}$

It has also become clear in recent years that the constant $E_{L 3}$ must be higher under stable stratification (Kantha, 1988; Bur- chard, 2001; Baumert and Peters, 2000). We will show that theoretical considerations dictate:

$E_{L 1}=2, \quad E_{L 2}=1$

and proper modeling of the diffusion term leads to $E_{L 4}=0$, thus removing a major objection to the use of the $q^{2} \ell$ equation.

\section{The $\mathrm{kT}(\mathbf{q l})$ equation}

Zeierman and Wolfshtein (1986) have derived an equation for what they call the time scale of turbulence rather rigorously, starting from an equation for the autocorrelation of the turbulence velocity

$R_{i j}(\boldsymbol{x}, t, \tau)=\overline{u_{j}(\boldsymbol{x}, \boldsymbol{t}) u_{j}(\boldsymbol{x}, t+\tau)}$

obtained by multiplying the Navier-Stokes equation for the turbulence component $u_{j}$ by $u_{j}(t+\tau)$ and adding the result to the product of the equation for $u_{j}(t+\tau)$ and $u_{j}(t)$ and ensemble-averaging. Under the assumption of quasistationarity, one obtains

$$
\frac{\mathrm{D}}{\mathrm{D} t} R_{i i}=-\left(R_{i j}+R_{j i}\right) \frac{\partial U_{i}}{\partial x_{j}}-\frac{1}{2} \frac{\partial}{\partial x_{j}}\left(S_{i j, i}+S_{i, i j}\right)-\frac{1}{\rho}\left(\frac{\partial K_{p, j}}{\partial x_{j}}+\frac{\partial K_{j, p}}{\partial x_{j}}\right)+v \frac{\partial^{2} R_{i i}}{\partial x_{j} \partial x_{j}}-2 v\left(\frac{\overline{\partial u_{j}(t)}}{\partial x_{j}} \frac{\partial u_{j}(t+\tau)}{\partial x_{j}}\right)
$$

where

$$
\begin{aligned}
S_{i j, k} & =\overline{u_{i}(t) u_{j}(t) u_{k}(t+\tau)}, \\
K_{p, j} & =\overline{p(t) u_{j}(t+\tau)}, \quad K_{j, p}=\overline{u_{j}(t) p(t+\tau)}
\end{aligned}
$$

are triple velocity and pressure-velocity correlations. Both the TKE equation and the time-scale equation can be derived from Eq. (22), the first by taking the limit $\tau=0$, and the second by integration with respect to $\tau$, since the TKE and the time scale are given by:

$$
\begin{aligned}
& k(\boldsymbol{x})=\frac{1}{2} R_{i i}(\boldsymbol{x}, \tau=0)=\frac{1}{2} \overline{u_{i} u_{i}} \\
& T(\boldsymbol{x})=\frac{1}{2 k} \int_{0}^{\infty} R_{i i}(\boldsymbol{x}, \tau) d \tau
\end{aligned}
$$

Eqation (22) after some closure assumptions can be written for a thin horizontal shear layer, after the inclusion of the buoyancy term, as an equation for $k T$ as

$$
\begin{aligned}
& \frac{\mathrm{D}}{\mathrm{D} t}(k T)-\frac{\partial}{\partial z}\left[\frac{v_{t}}{\sigma_{v}} \frac{\partial}{\partial z}(k T)\right]=P_{v}+B_{v}-\varepsilon_{v} \\
&=T\left\{C_{\nu 1}\left(-\overline{u w} \frac{\partial U}{\partial z}\right)+C_{\nu 3}(\beta g \overline{w \theta})-C_{\nu 2} \varepsilon\right\}
\end{aligned}
$$

where $T=C_{\mu} k^{2} / \varepsilon$. Zeierman and Wolfshtein (1986) chose

$C_{v 1}=0.173, \quad C_{v 2}=0.225$,

$\sigma_{v}=10.8$ and $\sigma_{k}=1.46$

They did not include the buoyancy term and therefore the value of $C_{v 3}$ is not known. Also the value of the $\sigma_{v}$ they chose based on the analysis of a high pressure gradient flow is too high. They also chose a traditional value for $C_{\mu}$ of 0.09 .

By substituting $T \sim \ell / q$, Eq. (25) can be converted to an equation for $q \ell$, a quantity proportional to the turbulent 
viscosity:

$$
\begin{aligned}
& \frac{\mathrm{D}}{\mathrm{D} t}(q \ell)-\frac{\partial}{\partial z}\left[q \ell S_{\ell v} \frac{\partial}{\partial z}(q \ell)\right]=P_{q \ell}+B_{q \ell}-\varepsilon_{q \ell} \\
& \quad=\frac{\ell}{q}\left\{E_{\nu 1}\left(-\overline{u w} \frac{\partial U}{\partial z}\right)+E_{\nu 3}(\beta g \overline{w \theta})-E_{\nu 2} \frac{q^{3}}{B_{1} \ell}\right\}
\end{aligned}
$$

Closure constants $E_{\nu 1}, E_{\nu 2}, E_{\nu 3}$, and $S_{\ell v}$ are related to $C_{\nu 1}$, $C_{v 2}, C_{v 3}$ and $\sigma_{v}$ by:

$$
\begin{aligned}
E_{\nu 1} & =2 C_{\nu 1}, \quad E_{\nu 2}=2 C_{\nu 2}, \quad E_{\nu 3}=2 C_{\nu 3}, \\
S_{\ell v} & =\frac{1}{\sigma_{T} B_{1}^{1 / 3}}
\end{aligned}
$$

so that

$E_{\nu 1}=0.346, \quad E_{\nu 2}=0.45$

We will show that from theoretical consideration, $E_{\nu 1}=1$, $E_{\nu 2}=0$. We will also show that the diffusion term, modeled as in Eqs. (25) and (27) leads to difficulties.

It should be noted that Nee and Kovasznay (1969) were the first to propose an equation for turbulent viscosity. Their approach was however purely empirical and they did not use it as an equation for the turbulence length scale.

\section{The $\omega(\mathbf{q} / \mathbf{l})$ equation}

Spalding (1969), Daly and Harlow (1970) and Saffman (1970) independently proposed a transport equation for mean vorticity fluctuations, which is proportional to $q^{2} / \ell^{2}$, and Ilegbusi and Spalding (1985) tried to improve it by removing the wall correction term. An appealing aspect of this equation is that it deals with a rather fundamental property of turbulence; it is the vorticity fluctuations resulting from the breakdown of vortex sheets and filaments into less ordered structures that give turbulence its characteristic chaotic structure. By dealing with such a fundamental property, one hopes to put more physical meaning into the model. The major drawback is however that the vorticity fluctuations are concerned with the property of small scales of turbulence. Although vortex stretching is a fundamental characteristic of turbulence, it is hard to see how an equation for a quantity dominated by small scales (which are asymptotically far removed from energy containing scales in the turbulence spectrum and therefore do not feel the large scales directly) can be used to describe the turbulence macroscale. Mellor's (1985) objection to the use of the dissipation equation is perhaps more valid here.

However if one interprets $q / \ell$ as an equation for turbulence frequency, as Kolmogoroff (1942) did, then a transport equation for this quantity is meaningful as a length scale equation. This approach has been developed further by Wilcox (1984, 1988a, 1988b, see also Wilcox and Traci, 1976). An acknowledged advantage of the $k-\omega$ model, compared to other models is that it can be integrated right through the viscous sublayer to the solid wall, without the need for wall functions. This has made it the workhorse of industrial applications. However, while this model has been known to perform well in adverse pressure gradients, it does suffer from a major disadvantage, namely its high sensitivity to the value of the free stream vorticity prescribed.

An equation for $\omega$ can be written for a thin horizontal shear layer as:

$$
\begin{aligned}
\frac{\mathrm{D}}{\mathrm{D} t}(\omega) & -\frac{\partial}{\partial z}\left[\frac{v_{t}}{\sigma_{\omega}} \frac{\partial}{\partial z}(\omega)\right]=P_{\omega}+B_{\omega}-\varepsilon_{\omega} \\
= & \frac{\omega}{k}\left\{C_{\omega 1}\left(-\overline{u w} \frac{\partial U}{\partial z}\right)+C_{\omega 3}(\beta g \overline{w \theta})-C_{\omega 2} \varepsilon\right\}
\end{aligned}
$$

Wilcox (1988) uses $C_{\omega 1}=5 / 9, C_{\omega 2}=5 / 6, \sigma_{\omega}=2$ and $\sigma_{k}=2$. He has not considered buoyancy terms and so the value of $C_{\omega 3}$ is unknown. Equation (30) can be converted readily to an equation for $q / \ell$ :

$$
\begin{aligned}
& \frac{\mathrm{D}}{\mathrm{D} t}\left(\frac{q}{\ell}\right)-\frac{\partial}{\partial z}\left[q \ell S_{\ell \omega} \frac{\partial}{\partial z}\left(\frac{q}{\ell}\right)\right]=P_{q / \ell}+B_{q / \ell}-\varepsilon_{q / \ell} \\
& =\frac{1}{q \ell}\left\{E_{\omega 1}\left(-\overline{u w} \frac{\partial U}{\partial z}\right)+E_{\omega 3}(\beta g \overline{w \theta})-E_{\omega 2} \frac{q^{3}}{B_{1} \ell}\right\}
\end{aligned}
$$

Closure constants $E_{\omega 1}, E_{\omega 2}, E_{\omega 3}$ and $S_{\ell \omega}$ are related to $C_{\omega 1}$, $C_{\omega 2}, C_{\omega 3}$ and $\sigma_{k T}$ by:

$$
\begin{aligned}
& E_{\omega 1}=2 C_{\omega 1}, \quad E_{\omega 2}=2 C_{\omega 2}, \quad E_{\omega 3}=2 C_{\omega 3} \\
& S_{\ell \omega}=\frac{1}{\sigma_{\omega} B_{1}^{1 / 3}}
\end{aligned}
$$

We will show that from theoretical considerations, $E_{\omega 1}=1$, $E_{\omega 2}=2$.

\section{The $T(1 / q)$ equation}

Speziale et al. (1990) argue that while $k-\omega$ model is computationally more robust than the $k-\varepsilon$ model for integration through the viscous layer to the solid boundary, it yields results for the TKE that are asymptotically inconsistent with theoretical results near a solid boundary. They show that this stems from the absence of a viscous cross-diffusion term in the $\omega$ equation. They suggest an alternative equation for the turbulence time scale $T=C_{\mu} k^{2} / \varepsilon$ (instead of frequency), which has the correct asymptotic behavior near the solid boundary. The form is similar to other length scale equations:

$$
\begin{aligned}
\frac{\mathrm{D} T}{\mathrm{D} t} & -\frac{\partial}{\partial z}\left[\frac{v_{t}}{\sigma_{T}} \frac{\partial}{\partial z}(T)\right]=P_{T}+B_{T}-\varepsilon_{T} \\
& =\frac{T}{k}\left\{C_{T 1}\left(-\overline{u w} \frac{\partial U}{\partial z}\right)+C_{T 3}(\beta g \overline{w \theta})-C_{T 2} \varepsilon\right\}
\end{aligned}
$$

with $C_{T 1}=-0.44$ and $C_{T 2}=-0.83$. Speziale et al. (1990) ignore the buoyancy term and so the value of $C_{T 3}$ is unknown. The asymptotic values for these constants should be -0.5 and -1.0 as shown in Sect. 8 .

Finally, it should be pointed out that an equation can also be written for the length scale $\ell$ itself that has a similar structure to the length scale equations detailed above, namely an equation involving shear and buoyancy production terms as well as the dissipation and diffusion terms. 


\section{The general length scale $\left(q^{m} l^{n}\right)$ equation}

Since the structure of all the length scale equations is the same, it is tempting to form an equation for a general quan- tity $q^{m} \ell^{n}$ from which the various length scale equations can be derived as subsets. Doing so, we get:

$$
\begin{aligned}
\frac{\mathrm{D}}{\mathrm{D} t}\left(q^{m} \ell^{n}\right)-\frac{\partial}{\partial z}\left[q \ell S_{\ell g} \frac{\partial}{\partial z}\left(q^{m} \ell^{n}\right)\right] & =P_{g}+B_{g}-\varepsilon_{g} \\
= & q^{m-2} \ell^{n}\left\{E_{1}\left(-\overline{u w} \frac{\partial U}{\partial z}\right)+E_{3}(\beta g \overline{w \theta})-E_{2} \frac{q^{3}}{B_{1} \ell}\left[1+E_{4}\left(\frac{\ell}{\kappa L}\right)^{2}\right]\right\}
\end{aligned}
$$

This equation must provide consistent length scale behavior in the constant flux region of a neutrally stratified flow near a solid wall. It can be shown (see Sect. 9) that this compatibility relation for Eq. (34) becomes

$$
-B_{1} \kappa^{2} S_{\ell g} n^{2}=E_{1}-E_{2}\left[1+E_{4}\right]
$$

To assure positive-definiteness of the diffusion coefficient, $S_{\ell g}$, the model constants $E_{1}$ and $E_{2}$ must be chosen such that the term on the right hand side is negative. For models with $E_{4}=0$, this means $E_{2}>E_{1}$. All the models discussed above satisfy this constraint, except the $q^{2} \ell$ model (Mellor and Yamada, 1982; Kantha and Clayson, 1994) and this is the reason $q^{2} \ell$ model cannot function without a non-zero value for $E_{4}$. In the $q \ell$ equation of Zeierman and Wolfshtein
(1986), $E_{1}$ and $E_{2}$ are too close and the resulting value for the diffusion coefficient is too small (0.039), or equivalently the value of the Prandtl number $\sigma_{\nu}$ is too large. We will show shortly that the asymptotically correct value for $E_{2}$ is 0 in the $q \ell$ model, and consequently, the diffusion coefficient $S_{\ell g}$ is always negative. Hence this model is physically untenable. Both the $\varepsilon$ and $\omega$ equations have sufficiently large values for $S_{\ell g}$ with $E_{4}=0$ and have enjoyed considerable success.

The problems with the $q^{2} \ell$ and $q \ell$ equations therefore stem from the diffusion term. This can be corrected as follows. Since the $\varepsilon$ equation performs well, combining the TKE Eq. (3) and the $q^{3} / \ell$ Eq. (10), we can derive an equation for $q^{m} \ell^{n}$ :

$$
\begin{aligned}
\frac{\mathrm{D}}{\mathrm{D} t}\left(q^{m} \ell^{n}\right)+D_{g} & =P_{g}+B_{g}-\varepsilon_{g} \\
& =q^{m-2} \ell^{n}\left\{\left[m+\left(3-E_{\varepsilon 1}\right) n\right](-\overline{u w}) \frac{\partial U}{\partial z}+\left[m+\left(3-E_{\varepsilon 3}\right) n\right](\beta g \overline{w \theta})-\left[m+\left(3-E_{\varepsilon 2}\right) n\right] \frac{q^{3}}{B_{1} \ell}\right\}
\end{aligned}
$$

where the form of the diffusion term $D_{g}$ is given by

$$
D_{g}=-q^{m-3} \ell^{n+1} \frac{\partial}{\partial z}\left[\frac{1}{q^{m-4} \ell^{n}} S_{\ell \varepsilon} \frac{\partial}{\partial z}\left(q^{m} \ell^{n}\right)\right]-\left(\frac{m+3 n}{2}\right) q^{m-2} \ell^{n}\left[\frac{\partial}{\partial z}\left(q \ell S_{q} \frac{\partial}{\partial z} q^{2}\right)-\frac{\ell}{q} \frac{\partial}{\partial z}\left(q^{2} S_{\ell \varepsilon} \frac{\partial}{\partial z} q^{2}\right)\right]
$$

If we rewrite Eq. (37) as

$$
\begin{aligned}
D_{g}=-\frac{\partial}{\partial z}\left[q \ell S_{\ell \varepsilon} \frac{\partial}{\partial z}\left(q^{m} \ell^{n}\right)\right]-q^{m-2} \ell^{n+2} S_{\ell \varepsilon} & \frac{\partial}{\partial z} \\
& \left(q^{3-m} \ell^{-n-1}\right) \frac{\partial}{\partial z}\left(q^{m} \ell^{n}\right) \\
& -\left(\frac{m+3 n}{2}\right) q^{m-2} \ell^{n}\left[\frac{\partial}{\partial z}\left(q \ell S_{q} \frac{\partial}{\partial z} q^{2}\right)-\frac{\ell}{q} \frac{\partial}{\partial z}\left(q^{2} S_{\ell \varepsilon} \frac{\partial}{\partial z} q^{2}\right)\right]
\end{aligned}
$$

where the first term is similar to the diffusion term in Eq. (34), it is clear that this term is not adequate to model diffusion correctly. The use of Eq. (37) eliminates the inconsistencies with the earlier length scale models and holds for any integer values of $m$ and $n$.

Umlauf and Burchard (2002) have also attempted to present a general length scale model, but with traditional form of the diffusion term as in Eq. (34). Consequently, their model works only when $n$ is a negative integer. This, among other things prompted them to suggest that a non-integer values be used for $n$. It is worth noting that while mathematically feasible, this approach is fraught with conceptual difficulties related to the physical meaning of the quantity $q^{m} \ell^{n}$ when $m$ and $n$ are not integers. For future reference, we will rewrite Eq. (36) in the form:

$$
\begin{aligned}
\frac{\mathrm{D}}{\mathrm{D} t}\left(q^{m} \ell^{n}\right)+D_{g}=P_{g}+B_{g}-\varepsilon_{g} & \\
& =q^{m-2} \ell^{n}\left\{E_{1}\left(-\overline{u w} \frac{\partial U}{\partial z}\right)+E_{3}(\beta g \overline{w \theta})-E_{2} \frac{q^{3}}{B_{1} \ell}\right\}
\end{aligned}
$$


where

$E_{1}=m+\left(3-E_{\varepsilon 1}\right) n, \quad E_{2}=m+\left(3-E_{\varepsilon 2}\right) n$

$E_{3}=m+\left(3-E_{\varepsilon 3}\right) n$

Closure constants in all previous length scale models can be obtained from Eq. (39) by a suitable choice for the integers $m$ and $\mathrm{n}$.

\section{Closure constants in $\mathbf{q}^{2}-\mathbf{q}^{\mathbf{m}} \ell^{\mathbf{n}}$ model}

We now appeal to well-known turbulent flows to evaluate the closure constants in this model.

\subsection{Tennekes hypothesis}

To determine the value of $E_{\varepsilon 1}$, we follow Baumert and Peters (2000) and derive an equation for $\partial \ell / \partial t$ using Eqs. (3) and (36). For a homogeneous shear flow far away from a solid surface, the diffusion term can be neglected and since

$\frac{1}{q^{m} \ell^{n}} \frac{\partial}{\partial t}\left(q^{m} \ell^{n}\right)=n\left(\frac{1}{\ell} \frac{\partial \ell}{\partial t}\right)+\frac{m}{2}\left(\frac{1}{q^{2}} \frac{\partial q^{2}}{\partial t}\right)$

we get

$$
\begin{aligned}
& \left(\frac{1}{\ell} \frac{\partial \ell}{\partial t}\right) \\
& \quad=\frac{1}{q^{2}}\left[\left(3-E_{\varepsilon 1}\right) P+\left(3-E_{\varepsilon 3}\right) B-\left(3-E_{\varepsilon 2}\right) \varepsilon\right]
\end{aligned}
$$

Tennekes (1989) noted that in a homogeneous shear flow, since the shear is homogeneous, it cannot impose a length scale and therefore, the shear term must drop out of the equation for the rate of change of turbulence length scale. This dictates that $E_{\varepsilon 1}=3$.

\subsection{Homogeneous, isotropic turbulence behind a grid}

To determine the value of $E_{\varepsilon 2}$, we appeal to homogeneous, isotropic turbulence in a neutrally stratified flow behind a grid in a wind tunnel. In this case, the diffusion and production terms can be neglected and Eqs. (3) and (36) reduce to:

$$
\begin{aligned}
& \frac{\partial q^{2}}{\partial t}=-2 \varepsilon \\
& \frac{\partial}{\partial t}\left(q^{m} \ell^{n}\right)=-q^{m-2} \ell^{n}\left[m+\left(3-E_{\varepsilon 2}\right) n\right] \varepsilon
\end{aligned}
$$

By assuming $q \sim t^{-p}$, it can be shown that $\ell \sim t^{1-p}$ and $E_{\varepsilon 2}=(2 p+1) / p$ from Eq. (42). While experiments in large wind tunnels (Gad-el-Hak and Corrsin, 1974; Comte-Bellot and Corrsin, 1966) show that the value of $p$ is between 0.5 and 0.65 , it must be noted that it is quite difficult to achieve the condition of homogeneity and isotropy accurately and large enough Reynolds numbers in the laboratory. Domaradzki and Mellor (1984) explain these departures from the $t^{-1}$ behavior that results from theoretical considerations which suggest that $p$ must be $1 / 2$ (precisely the value chosen by Mellor and Yamada, 1982). Choosing this value for $p$, we get $E_{\varepsilon 2}=4$.

\subsection{Constant flux region in neutrally stratified flows}

In the constant flux region of a steady neutrally stratified boundary layer, buoyancy terms are zero and simple dimensional reasoning leads to

$q=B^{1 / 3} u_{*}, \quad \ell=\kappa z, \quad \frac{\partial U}{\partial z}=\frac{u_{*}}{\kappa z}$

where $u_{*}$ is the friction velocity. Also $\partial q / \partial z=0 ; \partial / \partial t \equiv 0$. Neglecting tendency and buoyancy terms, and substituting these values, it can be shown that $q^{2}$ Eq. (3) is identically satisfied, irrespective of the value of $S_{q}$ or $B_{1}$ since the diffusion terms are identically zero and local equilibrium prevails with production balancing dissipation. Substitution in the $q^{m} \ell^{n}$ Eq. (36) gives:

$B_{1} \kappa^{2} S_{\ell \varepsilon}=E_{\varepsilon 2}-E_{\varepsilon 1}$

irrespective of the value of $m$ or $n$. Substituting the asymptotic values on the right hand side, we get $S_{\ell \varepsilon}=0.377$. This corresponds to a value for $\sigma_{\varepsilon}=1.041$ in $k-\varepsilon$ models, a value close to unity.

9.4 Turbulence generated by a stirring grid in a neutrally stratified fluid

Turbulence generated by an oscillating grid has been studied extensively in the laboratory both in neutrally and stably stratified fluids by turbulence researchers (Thompson and Turner, 1975; Hopfinger and Toly, 1976; Turner, 1981; E and Hopfinger, 1986; Nokes, 1988; Hannoun et al., 1988). These experiments provide considerable empirical knowledge on the behavior of turbulence in a particularly unique situation, when the only source of turbulence in regions far away from the grid is the turbulent diffusion. Thus these experiments provide a particularly useful and interesting data set for evaluating the modeling of diffusion terms in second moment closure models as suggested by Sonin (1983). Although it was feared that earlier such experiments (for example, Thompson and Turner, 1975) might have been contaminated by unwanted secondary circulations due to unavoidable asymmetries in the grid, it appears that these fears were greatly exaggerated. More recent experiments ( $E$ and Hopfinger, 1986, see also Hopfinger, 1987), with considerable attention given to eliminating secondary circulations, have in general confirmed earlier results. The experiments do suffer from a variety of problems, including wall effects, finite Reynolds numbers and finite oscillation amplitude, and the power law for the decay of TKE with distance from the source is not known precisely.

Dimensional considerations dictate that the turbulence length scale must scale as the distance from the grid midplane $z(\ell=b z)$, but the value of $p$ in $q^{2} \sim z^{-2 p}$ is quite uncertain (between 1 and 1.5). The situation here is somewhat analogous to decaying turbulence behind a grid in a wind 
tunnel, where theory dictates $q^{2} \sim t^{-1}$ in the ideal situation of infinite Reynolds number turbulence, while experimental values suggest a non-unity exponent in the necessarily nonideal experimental setups. Nevertheless, there are indications that $q \sim z^{-1}$ so that the dissipation rate $\varepsilon \sim z^{-4}$ and turbulent viscosity $v_{t} \sim$ constant.

E and Hopfinger (1986) found $b=0.24$ at sufficiently large values of grid stroke (and hence large Reynolds numbers) in agreement with Hopfinger and Linden (1982), although it is substantially less at lower values of grid strokes, between 0.1 and 0.2. Note that there is no apriori reason to assume that $b$ must be equal to $\kappa$ (in fact $\kappa$ is defined as the proportionality constant in the $\ell \sim z$ relationship in the logarithmic region of a neutrally-stratified boundary layer adjacent to a solid surface, and this has nothing to do with decaying turbulence behind a grid or for that matter turbulence generated by wave breaking).

Neglecting the tendency and buoyancy terms (production term is identically zero) and putting $q=a z^{-p}$ and $\ell=b z$, Eq. (3) gives:

$3 B_{1} b^{2} S_{q}=\frac{1}{p^{2}}$

whereas Eq. (36) gives

$3 B_{1} b^{2} S_{\ell \varepsilon}=\frac{(m+3 n)\left(3 B_{1} b^{2} S_{q}\right)-\left[m+\left(3-E_{\varepsilon 2}\right) n\right]}{n(3 p+1)(4 p+1)}$

Using Eq. (45), Eq. (46) becomes

$3 B_{1} b^{2} S_{\ell \varepsilon}=\frac{E_{\varepsilon 2}}{(3 p+1)(4 p+1)}$

independent of the values of $m$ and $n$. Dividing Eq. (47) by Eq. (45), we get

$$
\frac{S_{\ell \varepsilon}}{S_{q}}=\frac{3 p^{2} E_{\varepsilon 2}}{(3 p+1)(4 p+1)}
$$

independent of the value of $b$. For $p=1, S_{\ell \varepsilon} / S_{q}=0.6$ (and for $p=1.5, S_{\ell \varepsilon} / S_{q}=0.538$ ). Using the asymptotic value of $p=1$, since $S_{\ell \varepsilon}=0.377, S_{q}=0.628$. This is the value we choose; it corresponds to $\sigma_{k}$ of 0.624 , much lower than the traditional value of 1.0 in $k-\varepsilon$ models. Note also that $\sigma_{\varepsilon}=1.04$ and $S_{\ell \varepsilon} / S_{q}$ is not unity.

If we now substitute the value of $S_{q}$ in Eq. (45), we get a value for $b$ equal to 0.18 , well within the experimental range of values.

Lele (1985) was the first to argue that the diffusion coefficients in the TKE and the dissipation rate equations must not have the same value if there is to be a single turbulence front when a patch of turbulence spreads and diffuses into a quiescent fluid. This means that the $q^{2}$ and $\varepsilon$ fronts must propagate at the same speed. This relates the two diffusion coefficients (Lele 1985):

$$
\frac{S_{\ell \varepsilon}}{S_{q}}=6\left\{\left[1+\left(E_{\varepsilon 2}\right)^{2}\right]^{1 / 2}-E_{\varepsilon 2}\right\}
$$

For $E_{\varepsilon 2}=4$, this yields $S_{\ell \varepsilon} / S_{q}=0.739$, not far from the value given by Eq. (48) for $p=1$.
It should however be pointed out that Cazalbou et al. (1994) question Lele's analysis and have derived instead alternative constraints on $\sigma_{k}$ and $\sigma_{\varepsilon}: 4>2 \sigma_{k}>\sigma_{\varepsilon}$ and $2 \sigma_{k}=1+\sigma_{\varepsilon}$. The values we have chosen for $\sigma_{k}$ and $\sigma_{\varepsilon}$ do satisfy these constraints.

It is also worth noting that Umlauf and Burchard (2002) show that for traditional values of the closure constants, the $k-\varepsilon$ two-equation model cannot reproduce stirring grid data, predicting an excessive decay rate for TKE. This problem can be traced directly to the traditional value of $\sigma_{k}$ of around 1.0 used in their analysis. If the lower value derived above is used, this problem is eliminated.

\subsection{Turbulence generated by a grid in a stably stratified fluid}

The buoyancy term in the length scale equation is of great importance in geophysical flows, because of the ubiquitous presence and prominence of gravitational stratification. There is considerable uncertainty as to the value of the closure constant related to this term. For example, Rodi (1987) recommended a value of 0.2 for $C_{\varepsilon 3}$. It can be shown (see below) that $C_{\varepsilon 3}$ must assume negative values under stable stratification (or equivalently the constant $E_{L 3}$ in the $q^{2} \ell$ equation must be larger than $E_{L 1}$ ). Baumert and Peters (2000) indicate excellent agreement with observational results for a value of -1.4 for $C_{\varepsilon 3}$.

There exist a suite of experiments on the decay of gridgenerated turbulence in stably stratified fluids, starting from the pioneering experiments of Dickey and Mellor (1980), that show that there is an upper bound on the parameter $\tau=N \ell / q$, beyond which the decay of turbulence changes due to more efficient conversion of TKE into internal wave energy (Stillinger, Helland and Van Atta, 1983; Itsweire, Helland and Van Atta, 1986; see also Hopfinger, 1987; Browand, Guyomar and Yoon, 1987; and Rohr and Van Atta, 1987).

This upper bound on the length scale can also be derived from energetics arguments. If we assume that an eddy converts all its vertical kinetic energy $w^{2} / 2$ during overturning, the resulting gain in potential energy cannot exceed the vertical kinetic energy, or equivalently $N^{2} \ell^{2}<w^{2}$. The value of $w^{2} / q^{2}$ ranges from about 0.22 under neutral stratification to about 0.13 , when the flux Richardson number reaches its critical value for extinction of turbulence by stable stratification. This means that the upper bound on $\tau$ is between 0.47 and 0.36 . Whatever its precise value, the physical interpretation is clear.

The parameter $\tau$ is also the ratio of buoyancy frequency $N$ to the turbulence frequency (term coined by none other than Kolmogoroff, 1942) $q / \ell$. This means that as turbulence frequency approaches $\mathrm{N}$, efficient generation of internal waves by turbulent eddies becomes more and more feasible, so that energy in turbulence can be drained away rapidly by stable stratification. Clearly this ratio cannot exceed unity, since the frequency of internal waves is bounded by the buoyancy frequency. In practice, the turbulence frequency does not 
drop below about twice the buoyancy frequency, or equivalently the turbulence length scale does not exceed 1.4 times the Ozmidov length scale according to the observations of Stillinger et al. (1983) and Itsweire et al. (1986). Therefore, $\ell<1.4 L_{O}$, where $L_{O}=\left(\varepsilon / N^{3}\right)^{1 / 2}$ is the Ozmidov scale. This leads to an upper bound on $\tau$ of $1.4\left(B_{1}\right)^{1 / 2}$ or 0.49 since $B_{1}=16.6$ (Mellor and Yamada, 1982; Kantha and Clayson, 1994).

The upper bound on the turbulence length scale under stable stratification has been invoked in turbulence modeling by imposing an auxiliary condition on the model solutions that limits the length scale to values below that given by $\tau=c_{1}$ (Deardorff, 1973; Lewellen and Teske, 1973; Kantha, 1988; Galperin et al., 1988; Kantha and Clayson, 1994). The value of $c_{1}$ chosen by modelers to-date ranges between 0.2 and 0.6 ; Kantha and Clayson (1994) used a value of 0.53. However, it is possible to avoid imposing this auxiliary condition by choosing a proper value for $E_{L 3}$ (Kantha, 1988; Burchard, 2001).

To do so, we appeal to the experiments of Dickey and Mellor (1980), who raised a grid in a stably stratified fluid in a tank and measured the decay of turbulent fluctuations with time after the passage of the grid. They found that immediately after the passage of the grid, turbulence decayed as if there were no stratification, yielding the classical asymptotic decay law of turbulence behind a grid in neutrally stratified flows $\left(q \sim t^{-1 / 2}\right)$. However, as time progressed, the rate of decay of turbulence changed, presumably because of the conversion of TKE into internal waves. They found that as the TKE decreased, the upper bound on the parameter $\tau$ was about 0.6.

Following Kantha (1988), Baumert and Peters (2000), and Burchard and Deleersnijder (2001) the value of $E_{\varepsilon 3}$ in Eq. (36) under stable stratification can be determined as follows. Consider the case of a homogeneous shear-free turbulence in a stably stratified flow, so that the production and diffusion terms can be neglected. This situation corresponds to the experiments of Mellor and Dickey (1980). Since

$\frac{1}{q^{m} \ell^{n}} \frac{\partial}{\partial t}\left(q^{m} \ell^{n}\right)=\frac{1}{n}\left(\frac{1}{T} \frac{\partial T}{\partial t}\right)-\frac{m+n}{2 n}\left(\frac{1}{q^{2}} \frac{\partial q^{2}}{\partial t}\right)$

where $T=\ell / q$, an equation can be derived for the turbulence time scale $T$ using Eqs. (3) and (36):

$\frac{1}{T} \frac{\partial T}{\partial t}=\frac{1}{q^{2}}\left[\left(2-E_{\varepsilon 3}\right) B-\left(2-E_{\varepsilon 2}\right) \varepsilon\right]$

Invoking the condition of equilibrium and setting $\partial T / \partial t=0$ when $T$ hits the upper bound $\tau_{u}$,

$\frac{E_{\varepsilon 3}-2}{E_{\varepsilon 2}-2}=\frac{\varepsilon}{B}$

The quasi-equilibrium second moment closure of Kantha and Clayson (1994) yields

$S_{H}=\frac{A_{2}\left[1-\left(6 A_{1} / B_{1}\right)\right]}{1+3 A_{2}\left[6 A_{1}+B_{2}\left(1-C_{3}\right)\right] \tau_{u}^{2}}$ for the stability coefficient $S_{H}$ in the equation for buoyancy production $B=-q \ell N^{2} S_{H}$. Therefore Eq. (52) can be written as

$$
\begin{aligned}
E_{\varepsilon 3}=2- & \left(E_{\varepsilon 2}-2\right) \\
& \left\{\frac{1+3 A_{2}\left[6 A_{1}+B_{2}\left(1-C_{3}\right)\right] \tau_{u}^{2}}{A_{2}\left(B_{1}-6 A_{1}\right) \tau_{u}^{2}}\right\}
\end{aligned}
$$

Using Kantha and Clayson (1994) values of $A_{1}=0.92$, $A_{2}=0.74, B_{1}=16.6, B_{2}=10.1$, and $C_{3}=0.2$, and $E_{\varepsilon 2}=4$, we get $E_{\varepsilon 3}=-6$ for $\tau_{u}=0.6$ (Dickey and Mellor, 1980). $E_{\varepsilon 3}$ is relatively insensitive to the exact value of $\tau_{u}$ as long as it is around 0.5 .

Using the values of $A_{1}=0.58, A_{2}=0.62, B_{1}=16.6$, $B_{2}=12.0$, and $C_{3}=0.2$ as recently updated by Kantha (2003), we get $E_{\varepsilon 3}=-4.66$ for $\tau_{u}=0.6$. This corresponds to $C_{\varepsilon 3}=-2.33$, close to the value of -2.1 indicated by Burchard and Baumert (1995) and Baumert and Peters (2000) to yield excellent agreement with observational results on mixing in the North Sea. We therefore choose $E_{\varepsilon 3}=-4.7$.

Burchard (2001) uses a slightly different approach to fixing the value of $C_{\varepsilon 3}$. By assuming local equilibrium and therefore neglecting tendency and diffusion terms in Eqs. (3) and (36), and substituting for the shear production term $P$ in Eq. (36) from Eq. (3), it can be shown that instead of Eq. (52), one gets

$\frac{E_{\varepsilon 3}-E_{\varepsilon 1}}{E_{\varepsilon 2}-E_{\varepsilon 1}}=\frac{\varepsilon}{B}$

so that Eq. (54) becomes

$$
\begin{aligned}
& E_{\varepsilon 3}=E_{\varepsilon 1} \\
& \quad-\left(E_{\varepsilon 2}-E_{\varepsilon 1}\right)\left\{\frac{1+3 A_{2}\left[6 A_{1}+B_{2}\left(1-C_{3}\right)\right] \tau_{u}^{2}}{A_{2}\left(B_{1}-6 A_{1}\right) \tau_{u}^{2}}\right\}
\end{aligned}
$$

For the Dickey and Mellor (1980) value of $\tau_{u}=0.6$, using the Kantha and Clayson (1994) values, we get $E_{\varepsilon 3}=-1$. But the updated Kantha (2003) values give $E_{\varepsilon 3}=-0.33$. This corresponds to $C_{\varepsilon 3}=-0.17$, and $E_{\varepsilon 3}=5.17$, close to the $E_{\varepsilon 3}$ value of 5.09 used by Burchard (2001).

However, there is a fundamental problem in using the Burchard (2001) approach. The experiment of Dickey and Mellor (1980) involved no shear and hence shear production $P$ was identically zero. The experiment never reached a steady state, but TKE continued to decrease after stable stratification asserted itself and put an upper bound on $\tau$. Therefore their experimental value of $\tau_{u}$ cannot be used to ascertain the value of $E_{\varepsilon 3}$ in this manner.

It is also worth noting that the TKE Eq. (3) under local equilibrium conditions can be rewritten as:

$\frac{\varepsilon}{B}=1-\frac{1}{R f}$

where $R f$ is the flux Richardson number. For the maximum possible value of $1 / 4$ for $R f$, beyond which turbulence is quenched, $\varepsilon / B=-3$. This value gives $E_{\varepsilon 3}=0$ using Eq. (55)! 
This is untenable. Using Eq. (52), one gets $E_{\varepsilon 3}=-4$ or equivalently $C_{\varepsilon 3}=-2.0$, close to the value derived above as well as the value -2.1 cited by Burchard and Baumert (1995) and Baumert and Peters (2000). It could be argued that $E_{\varepsilon 3}$ should be selected this way. However, the value of -4 for $E_{\varepsilon 3}$ obtained this way is quite close to the value of -4.7 we have chosen.

9.6 Turbulence at the center of a duct in a neutrally stratified fluid

Zeierman and Wolfshtein (1986) cite and use data from a channel (Clark, 1968; Laufer, 1950) to suggest a value for $\sigma_{k}$. For completeness, we cite these data, even though we suspect that it underestimates the value of $S_{q}$ or equivalently overestimates the value of $\sigma_{k}$. At the center of a channel, various turbulence quantities must be even functions of the distance $y$ from the axis. Experimental data suggest that the mean velocity and the TKE obey:

$$
\frac{U}{U_{0}}=1-0.242\left(\frac{z}{h}\right)^{2} ; \quad \frac{k}{k_{0}}=1+6.67\left(\frac{z}{h}\right)^{2}
$$

where $2 h$ is the width of the channel. The shear stress at the wall is

$\tau_{w}=0.048 k_{0}^{1 / 2} U_{0}$

By expanding $k$ and $\varepsilon$ as even functions of $(z / h)$ and making use of the fact that the shear stress varies linearly:

$\tau=v_{t} \frac{\partial U}{\partial z}=-\tau_{w}\left(\frac{z}{h}\right)$

it is possible to show that

$$
\begin{aligned}
& \frac{U}{U_{0}}=1-\left(\frac{\tau_{w} \varepsilon_{0} h}{2 C_{\mu} k_{0}^{2} U_{0}}\right)\left(\frac{z}{h}\right)^{2} ; \\
& \frac{k}{k_{0}}=1+\left(\frac{\sigma_{k} \varepsilon_{0} h^{2}}{2 C_{\mu} k_{0}^{3}}\right)\left(\frac{z}{h}\right)^{2}
\end{aligned}
$$

Equating quantities in Eqs. (58) and (61) and making use of Eq. (59): we get $C_{\mu} \sigma_{k}=0.13$, thus leading to a value of $\sigma_{k}=1.46$ and $S_{q}$ of 0.269 .

Since it is difficult to guess the likely values of the experimental constants in the channel in the asymptotic limit of infinite Reynolds numbers, we cannot guess the asymptotic values for $\sigma_{k}$ and $S_{q}$. We therefore reluctantly ignore this set of experiments.

Zeierman and Wolfshtein (1986) cite and use data from a high pressure gradient boundary layer to derive a value for $\sigma_{\varepsilon}$. It can be shown that for this flow

$$
\left(S_{\ell \varepsilon}-\frac{3}{2} E_{\varepsilon 1} S_{q}\right)\left(S_{\ell \varepsilon}-\frac{3}{2} E_{\varepsilon 2} S_{q}\right)=\frac{4\left(E_{\varepsilon 2}-E_{\varepsilon 1}\right)^{2}}{B_{1}^{2} K_{0}^{4}}
$$

where $K_{0}$ has an observed value of 0.48 (Townsend, 1961). Using the values of other constants, $S_{q}=0.174$, once again a small value consistent with the very high value derived by Zeierman and Wolfshtein (1986) for $\sigma_{\varepsilon}$ of 10.8. Since, once again it is hard to guess the asymptotic value for $K_{0}$, we reluctantly ignore this experimental data also.

With the values of closure constants determined as above for the general length scale equation, it is possible to evaluate their corresponding values for its various subsets, namely different closures, using Eq. (39):

$$
\begin{aligned}
k-\varepsilon\left(q^{3} / \ell ; m=3, n=-1\right): & E_{\varepsilon 1}=3, E_{\varepsilon 2}=4, E_{\varepsilon 3}=-4.7 \\
k-\omega(q / \ell ; m=1, n=-1): & E_{\omega 1}=1, E_{\omega 2}=2, E_{\omega 3}=-6.7 \\
q-q^{2} \ell(m=2, n=1): & E_{L 1}=2, E_{L 2}=1, E_{L 3}=9.7 \\
k-k T(q \ell ; m=1, n=1): & E_{\nu 1}=1, E_{\nu 2}=0, E_{\nu 3}=8.7 \\
k-T(\ell / q ; m=-1, n=1): & E_{T 1}=-1, E_{T 2}=-2, E_{T 3}=6.7 \\
q-\ell(m=0, n=1): & E_{\ell 1}=0, E_{\ell 2}=-1, E_{\ell 3}=7.7
\end{aligned}
$$

These values along with the values of $S_{q}$ and $S_{\ell \varepsilon}$ enable any length scale model to be used consistently and equivalently to others.

The closure constants can also be used to show why the $k-\varepsilon$ and $k-\omega$ have enjoyed a degree of success that $q-q^{2} \ell$ and k-kT have not. The constant flux region compatibility relationship Eq. (35) for the traditional form of the diffusion term can be rewritten as:

$$
\begin{aligned}
-B_{1} \kappa^{2} S_{\ell g} n^{2}=[m+ & \left.\left(3-E_{\varepsilon 1}\right) n\right] \\
& -\left[m+\left(3-E_{\varepsilon 2}\right) n\right]\left(1+E_{4}\right)
\end{aligned}
$$

When $E_{4}=0$, this becomes $B_{1} \kappa^{2} S_{\ell g}=-1 / n$, independent of the value of $m$, so that to avoid a physically absurd negative value for $S_{\ell g}, n$ must be negative. Only the $k-\varepsilon$ and $k-\omega$ models satisfy this requirement. Mellor and Yamada (1982) overcome this problem by using a nonzero positive value for $E_{4}$. Since the second term in Eq. (64) vanishes for the k-kT model, no value of $E_{4}$ can assure a positive value for $S_{\ell g}$.

\section{Influence of rotation on the length scale}

While the influence of the rotation of the reference frame on small scale turbulence is negligible in geophysical flow situations, this is not true for many engineering devices (Johnston et al. 1972, Watmuff et al. 1985). Also, it is not clear that rotation does not influence the large scale eddies. In fact, the maximum eddy size appears to be much smaller in rotating boundary layers such as the neutral atmospheric boundary layer (ABL) compared to nonrotating boundary layers in the laboratory. The length scale $\ell$ is about 0.09 times the boundary layer thickness in laboratory boundary layers (Mellor 1985), while a more appropriate value is 0.03 for the neutral ABL (recall the value of $\alpha$ in Eq. 6). The lack of rotational influence on dissipation and therefore on the length scale was cited at the 1980-1981 Stanford Conference on Complex Turbulent Flows as a major drawback of all second-moment closure models. Without explicit rotational terms in the length scale equation, it is impossible to reproduce the experimentally observed effect of rotation on turbulence decay. Wigeland and Nagib (1978) observed that when the turbulence behind a grid in a wind-tunnel is subjected to 
strong rotation, its dissipation rate altered substantially. Similar effects were also observed by Bardina et al. (1985) in their large eddy simulations of turbulence decay, which led them to suggest a modification for the $\varepsilon$ equation to account for the effect of rotation on grid-generated turbulence behind a grid, by the addition of a rotational term:

$\frac{\mathrm{d} \varepsilon}{\mathrm{d} t}=C_{\varepsilon 4} f \varepsilon-\frac{\varepsilon}{k}\left(C_{\varepsilon 2} \varepsilon\right)$

where $f=2 \Omega$ and $\Omega$ is the angular velocity of rotation. Solving this along with the corresponding TKE equation (note that reference frame rotation cannot affect the energy equation)

$\frac{\mathrm{d} k}{\mathrm{~d} t}=-2 \varepsilon$

we get

$k=k_{0}\left\{1+\frac{\varepsilon_{0}}{k_{0}} \frac{\left(C_{\varepsilon 2}-1\right)}{C_{\varepsilon 4} f}\left[1-\exp \left(-C_{\varepsilon 4} f t\right)\right]\right\}^{1 /\left(C_{\varepsilon 2}-1\right)}$

$$
\begin{aligned}
\varepsilon=\varepsilon_{0} e^{-C_{\varepsilon 4} t} & \left\{1+\frac{\varepsilon_{0}}{k_{0}} \frac{\left(C_{\varepsilon 2}-1\right)}{C_{\varepsilon 4} f}\left[1-\exp \left(-C_{\varepsilon 4} f t\right)\right]\right\}^{1 /\left(C_{\varepsilon 2}-1\right)}(68)
\end{aligned}
$$

where subscript 0 refers to initial values. The classical decay law for grid-generated turbulence becomes $q \ell=q_{0} \ell_{0} e^{C_{\varepsilon 4} f t}$ instead of $q \ell=$ constant.

Bardina et al. (1985) chose $C_{\varepsilon 2}=11 / 6$ and found that $C_{\varepsilon 4}=0.075$ reproduced experimental results and also agreed with their own LES results. However choosing the asymptotic value for $C_{\varepsilon 2}$ of 2 gives $C_{\varepsilon 4}=0.04$ and also yields the classical asymptotic decay law $k \sim t^{-1}$ when $f=0$.

While geophysical applications have seldom considered the explicit rotational terms, for completeness, we write down the form of Eq. (36) resulting when the rotational effects are incorporated:

$$
\begin{aligned}
& \frac{\mathrm{D}}{\mathrm{D} t}\left(q^{m} \ell^{n}\right)+D_{g}=P_{g}+B_{g}-\varepsilon_{g} \\
& \quad=q^{m-2} \ell^{n}\left\{\left[m+\left(3-E_{\varepsilon 1}\right) n\right]\left(-\overline{u w} \frac{\partial U}{\partial z}\right)+E_{\varepsilon 4} n|f| q^{2}+\left[m+\left(3-E_{\varepsilon 3}\right) n\right](\beta g \overline{w \theta})-\left[m+\left(3-E_{\varepsilon 2}\right) n\right] \frac{q^{3}}{B_{1} \ell}\right\}
\end{aligned}
$$

where $E_{\varepsilon 4}=C_{\varepsilon 4}=0.04,|f|=\sqrt{f_{i} f_{i}}=2 \Omega_{E}, \Omega_{E}$ being the angular rotation rate of the planet. This equation is also useful in the simulation of turbulence in engineering devices with high rotation rates. Note that only the effect of rotation on small scales of turbulence is taken into account in Eq. (69).

\section{Wall damping functions}

In many applications, it may be necessary to carry out calculations on mixing all the way to the boundary. Sediment transport in an oceanic bottom boundary layer is a typical example. In the calculation of turbulent flows involving separation, it is also necessary to integrate to the wall. To enable this, the eddy viscosity $v_{t}$, and the dissipation term in the $k-\varepsilon$ equations must be multiplied by wall damping functions (Speziale et al. 1990), or equivalently $C_{\mu}$ must be multiplied by $f_{\nu}$, and $C_{\varepsilon 2}$ by $f_{\varepsilon}$, where $f_{\nu}$ and $f_{\varepsilon}$ are wall damping functions. Speziale et al. (1990) recommend:

$$
\begin{aligned}
& f_{v}=\left[1+3.45\left(R e_{t}\right)^{-1 / 2}\right] \tanh \left(y^{+} / 70\right) \\
& f_{\varepsilon}=\left\{1-\frac{2}{9} \exp \left[-\left(\frac{R e_{t}}{6}\right)^{2}\right]\right\}\left[1-\exp \left(\frac{-y^{+}}{4.9}\right)\right]^{2}
\end{aligned}
$$

where $R e_{t}=k^{2} /(\nu \varepsilon)$ is the turbulence Reynolds number and $y^{+}=y u_{*} / \nu$. Functions $f_{\nu}$ and $f_{\varepsilon}$ have the correct asymptotic behavior needed near the wall: $f_{v} \sim O(1 / y) ; f_{\varepsilon} \sim O\left(y^{2}\right)$. Far from the wall, both $f_{\nu}, f_{\varepsilon} \rightarrow 1$.

In addition, viscous terms must be retained so that Eq. (3) becomes:

$$
\begin{gathered}
\frac{\mathrm{D}}{\mathrm{D} t}\left(q^{2}\right)-\frac{\partial}{\partial z}\left[\left(v+q \ell S_{q} f_{v}\right) \frac{\partial}{\partial z}\left(q^{2}\right)\right]=2(p+B-\varepsilon) \\
=-2 \overline{u w} \frac{\partial U}{\partial z}+2 \beta g \overline{w \theta}-2 \frac{q^{3}}{B_{1} \ell}+v \frac{\partial^{2}}{\partial z^{2}}\left(q^{2}\right)
\end{gathered}
$$

Equation (36) assumes the form:

$$
\begin{aligned}
\frac{\mathrm{D}}{\mathrm{D} t}\left(q^{m} \ell^{n}\right)+ & D_{g}=P_{g}+B_{g}-\varepsilon_{g}+V_{g} \\
= & q^{m-2} \ell^{n}\left\{\left[m+\left(3-E_{\varepsilon 1}\right) n\right]\left(-\overline{u w} \frac{\partial U}{\partial z}\right)+\left[m+\left(3-E_{\varepsilon 3}\right) n\right] \beta g \overline{w \theta}-\left[m+\left(3-E_{\varepsilon 2}\right) n\right] f_{\varepsilon} \frac{q^{3}}{B_{1} \ell}\right\} \\
& +v q^{m-2} \ell^{n}\left\{\frac{(m+3 n)}{n} \frac{1}{q^{m} \ell^{n}} \frac{\partial}{\partial z}\left(q^{m} \ell^{n}\right) \frac{\partial}{\partial z}\left(q^{2}\right)-\frac{(m+3 n)(m+n)}{4 n} q^{2}\left[\frac{1}{q^{2}} \frac{\partial}{\partial z}\left(q^{2}\right)\right]^{2}\right. \\
& \left.\quad-\frac{(n+1)}{n} q^{2}\left[\frac{1}{q^{m} \ell^{n}} \frac{\partial}{\partial z}\left(q^{m} \ell^{n}\right)\right]^{2}\right\}+v \frac{\partial^{2}}{\partial z^{2}}\left(q^{m} \ell^{n}\right)
\end{aligned}
$$


where

$$
\begin{aligned}
D_{g}=-q^{m-3} \ell^{n+1} \frac{\partial}{\partial z} & {\left[\left(v+\frac{f_{v}}{q^{m-4} \ell^{n}} S_{\ell \varepsilon}\right) \frac{\partial}{\partial z}\left(q^{m} \ell^{n}\right)\right] } \\
& -\left(\frac{m+3 n}{2} q^{m-2} \ell^{n}\right)\left\{\frac{\partial}{\partial z}\left[\left(v+q \ell f_{v} S_{q}\right) \frac{\partial}{\partial z} q^{2}\right]-\frac{\ell}{q} \frac{\partial}{\partial z}\left[\left(v+q^{2} f_{v} S_{\ell \varepsilon}\right) \frac{\partial}{\partial z} q^{2}\right]\right\}
\end{aligned}
$$

Note the presence of viscous cross-diffusion terms in Eq. (72). The absence of such a cross- diffusion term in the conventional $k-\omega$ model of Wilcox (1988) makes it yield asymptotically inconsistent solutions for near wall turbulence as indicated by Speziale et al. (1990). The crossdiffusion terms are absent in $k-\varepsilon$ model and it has the correct asymptotic behavior near the wall. The k-T model of Speziale et al. (1990) includes two nonzero cross diffusion terms to yield the correct asymptotic behavior. In any case, the presence of cross-diffusion terms and the damping functions makes the general $q^{m} \ell^{n}$ model have the correct asymptotic near-wall behavior for all values of $m$ and $n$. However, as Speziale et al. (1990) noted, the need to specify the boundary condition for the quantity being computed makes the k-T model more attractive than the $k-\omega$ model, since $T \sim 1 / \omega$ is not singular at the wall. The $k-\varepsilon$ model suffers from difficulties in deriving a proper value for $\varepsilon$ at the wall (the Neumann boundary condition of vanishing $\partial \varepsilon / \partial z$ is incorrect). Therefore, it may be preferable to use the k-T model when integrating down to the boundary.

\section{Concluding remarks}

We have shown that the traditional model for the diffusion term in the length scale equation is responsible for the necessity in the Mellor and Yamada (1982) $q^{2}-q^{2} \ell$ model for the arbitrary wall correction term and the inconsistent behavior of k-kT models. We have derived a general length scale equation that is devoid of such difficulties and from which any current length scale model can be derived as a subset. We have also derived the closure constants in this equation and indicated the forms of this equation under the influence of rotation and for near-wall turbulence calculations. We believe that the use of this general length scale equation (Eqs. 36 and 37) or one of its subsets (see Eq. 63) in two-equation models of turbulence will therefore be worthwhile in the future. We also recommend the use of the updated values for the second moment closure constants that occur in the stability functions of momentum and temperature, $S_{M}$ and $S_{H}$, as indicated by Kantha (2003), since they appear to overcome some of the deficiencies of earlier values used in Mellor and Yamada (1982), Kantha and Clayson (1994) and similar Mellor-Yamada type second moment closure models, as was shown first by Cheng et al. (2002).

Acknowledgements. We acknowledge with pleasure the partial support by the ONR under Grants N00014-99-1-0788, N00014-02-11043 and N00014-03-1-0488.
Edited by: S. Wiggins

Reviewed by: G. Mellor

\section{References}

Bardina, J., Ferziger, J. H., and Rogallo, R. S.: Effect of rotation on isotropic turbulence: Computation and modeling, J. Fluid Mech., 154, 321-336, 1985.

Baumert, H. and Peters, H.: Second-moment closures and length scales for weakly stratified turbulent shear flows, J. Geophys. Res., 105, 6453-6468, 2000.

Blackadar, A. K.: The vertical distribution of wind and turbulence exchange in a neutral atmosphere, J. Geophys. Res., 67, 30953102, 1962.

Bradshaw, P.: Understanding and prediction of turbulent flows, Aeronaut. J., 76, 403-418, 1972.

Browand, F. K., Guyomar, D., and Yoon, S.-C.: The behavior of a turbulent front in a stratified fluid: Experiments with an oscillating grid, J. Geophys. Res., 92, 5329-5341, 1987.

Burchard, H.: On the $q^{2} l$ equation by Mellor and Yamada (1982), J. Phys. Oceanogr., 31, 1377-1387, 2001.

Burchard, H. and Baumert, H.: On the performance of a mixedlayer model based on the $k-\varepsilon$ turbulence closure, J. Geophys. Res. 100, 8523-8540, 1995.

Burchard, H. and Bolding, K.: Comparative analysis of four second-moment closure models for the oceanic mixed layer, J. Phys. Oceanogr., 31, 1943-1968, 2001.

Burchard, H. and Deleersnijder, E.: Investigating the stability of algebraic non-equilibrium second-moment turbulence closure models, Ocean Modeling, 3, 33-50, 2001.

Cazalbou, J. B., Spalart, P. R., and Bradshaw, P.: On the behavior of two-equation models at the edge of a turbulent region, Phys. Fluids, A6, 1797-1804.

Cheng, Y. and Canuto, V. M.: Stably stratified shear turbulence: A new model for the energy dissipation scale, J. Atmos. Sci., 51, 2384-2396, 1994.

Cheng, Y., Canuto, V. M., and Howard, A. M.: An improved model for the turbulent PBL, J. Atmos. Sci., 59, 1550-1565, 2002.

Clark, J. A.: A study of incompressible turbulent boundary layers in channel flows, Trans. Amer. Soc. Mech. Engineers Paper 68FE-26, 1968.

Comte-Bellot, J. and Corrsin, S.: The use of a contraction to improve the isotropy of grid- generated turbulence, J. Fluid Mech., 25, 657-682, 1966.

Corrsin, S.: Limitations of gradient transport models in random walks and in turbulence, Adv. Geophys., 18A, 25-71, 1974.

Daly, B. J. and Harlow, F. H.: Transport equations in turbulence, Phys. Fluids., 13, 2634-2649, 1970.

Dickey, T. D. and Mellor, G. L.: Decaying turbulence in neutral and stratified fluids, J. Fluid Mech., 99, 13-31, 1980. 
Domaradzki, J. A. and Mellor, G. L.: A simple turbulence closure hypothesis for the triple-velocity correlation functions in homogeneous isotropic turbulence, J. Fluid Mech., 140, 45-61, 1984.

E, X. and Hopfinger, E. J.: On mixing across an interface in stably stratified fluid, J. Fluid Mech., 166, 227-234, 1986.

Gad-el-Hak, M. and Corrsin, S.: Measurement of nearly isotropic turbulrnce behind a uniform jet grid, J. Fluid Mech., 62, 115143, 1974.

Gibson, M. M. and Launder, B. E.: On the calculation of horizontal turbulent free shear flow under gravitational influence, ASME J. Heat Transfer, 98C, 81-87, 1976.

Galperin, B. and Hassid, S.: A modified turbulent energy model for geophysical flows: Influence of ground proximity, Bound- Layer Meteor., 35, 155-165, 1986.

Galperin, B., Kantha, L. H., Hassid, S., and Rosati, A.: A quasiequilibrium turbulent energy model for geophysical flows, J. Atmos. Sci., 45, 55-62, 1988.

Hanjalic, K. and Launder, B. E.: A Reynolds stress model of turbulence and its application to thin shear flows, J. Fluid Mech., 52, 609-638, 1972

Harlow, F. H., and Nakayama, P. I.: Turbulence transport equations, Phys. Fluids, 10, 2323-2423, 1967.

Hopfinger, E. J.: Turbulence in stratified fluids: A review, J. Geophys. Res., 92, 5287-5303, 1987.

Hopfinger, E. J. and Linden, P. F.: Formation of thermoclines in zero-mean-shear turbulence subjected to a stabilizing buoyancy flux, J. Fluid Mech., 114, 157-173, 1982.

Hopfinger, E. J. and Toly, J. A.: Spatially decaying turbulence and its relation to mixing across a density interface, J. Fluid Mech., 78, 155-175, 1976.

Ilegbusi, J. O. and Spalding, D. B.: An improved version of the $k-\omega$ model of turbulence, ASME J. Heat Transfer, 107, 63-69, 1985.

Itsweire, E. C., Helland, K. N., and van Atta, C. W.: The evolution of grid-generated turbulence in stably stratified fluid, J. Fluid Mech., 162, 299-338, 1986.

Johnston, J. P., Halleen, R. M., and Lazius, D. K.: Effects of spanwise rotation on the structure of two-dimensional fullydeveloped turbulent channel flow, J. Fluid Mech., 56, 533-557, 1972.

Kantha, L. H.: On some aspects and applications of second-moment closure, Atmospheric and Oceanic Sciences Program Report, Princeon University, Princeton, 167, 1988.

Kantha, L. H.: On an improved model for the turbulent PBL, J. Atmos. Sci. (in press), 2003.

Kantha, L. H., and Clayson, C. A.: An improved mixed layer model for geophysical applications, J. Geophys. Res., 99, 25235 25 266, 1994.

Kolmogoroff, A. N.: Equations of turbulent motion of an incompressible fluid, Izvest. Akad. Nauk. USSR, Ser. Phys., 6, 56-58, 1942.

Laufer, J.: Some recent measurements in a two-dimensional turbulent channel, J. Aeronaut. Sci., 17, 277-287, 1950.

Launder, B. E.: On the effects of gravitational field on the turbulent transport of heat and momentum, J. Fluid Mech., 67, 569-581, 1975.

Launder, B. E., Reece, G. J., and Rodi, W.: Progress in the development of Reynolds-stress closure, J. Fluid Mech., 68, 537-566, 1975.

Lele, S. K.: A consistency condition for Reynolds stress closures, Phys. Fluids, 28, 64-68, 1985.

Lewellen, W. S.: Use of invariant modeling, in: Handbook of Tur- bulence, edited by Frost, W. and Moulden, T. H., Plenum Press, 237-280, 1977.

Lewellen, W. S. and Teske, M.: Prediction of the Monin-Obukhov similarity functions from an invariant model of turbulence, J. Atmos. Sci., 30, 1340-1345, 1973.

Markatos, N. C.: The mathematical modeling of turbulent flows, Appl. Math. Modeling, 10, 190-220, 1986.

Martin, P. J.: Simulation of the mixed layer at OWS November and Papa with several models, J. Geophys. Res., 90, 903-916, 1985.

Martin, P. J.: Testing and comparison of several mixed layer models, NORDA Report143, US Navy, 30, 1986.

Mellor, G. L.: Ensemble average turbulence closure, Adv. Geophys., 28B, 345-358, 1985.

Mellor, G. L. and Herring, H. J.: A survey of mean turbulent field closure models, AIAA J.,11, 590-599, 1973.

Mellor, G. L. and Yamada, T.: Development of a turbulence closure model for geophysical fluid problems, Rev. Geophys. Space Phys., 20, 851-875, 1982.

Mellor, G. L. and Durbin, P. A.: The structure and dynamics of the ocean surface mixed layer, J. Phys. Oceanogr., 5, 718-728, 1975.

Nee, V. W. and Kovasznay, L. S. G.: Simple phenomenological theory of turbulent shear flows, Phys. Fluids, 16, 738-743, 1969.

Naot, D., Shavit, A., and Wolfshtein, M.: Two-point correlation model and the redistribution of Reynolds stresses, Phys. Fluids, 16, 738-743, 1973.

Nokes, R. I.: On the entrainment rate across a density interface, J. Fluid Mech., 188, 185-204, 1988.

Prandtl, L.:Über ein neues Formel-system für die ausgebildete Turbulenz, Nachr. Akad. Wiss. Göttingen, Math.-Phys.K1. 1945, 6 19,1945

Rodi, W.: Turbulence models for environmental problems, In Prediction Methods for Turbulent Flows, ed. by Kollmann, W. Hemisphere Publ. Co., 259-349, 1980.

Rodi, W.: Examples of calculation methods for flow and mixing in stratified fluids, J. Geophys. Res., 92, 5305-5328, 1987.

Rohr., J. and Van Atta, C.: Mixing efficiency in stably stratified growing turbulence. J. Geophys. Res., 92, 5481-5488, 1987.

Rotta, J. C.: Statistische Theorie nichthomogener Turbulenz, 1. Z. Phys., 129, 547-572, 1951.

Rotta, J. C.: Turbulente Strömungen, Teubner Press, Germany, 1972.

Saffman, P. G.: A model for inhomogeneous turbulent flow, Proc. Roy. Soc. London, A317, 417-433, 1970.

Sonin, A. A.: Calibration of the $k-\varepsilon$ turbulence model for the diffusion of turbulence, Phys. Fluids, 26, 2769, 1983.

Spalding, D. B.: The prediction of two-dimensional steady, turbulent elliptic flows, International Seminar on Heat and Mass Transfer with Separated Regions, Yugoslavia, Sept. 1-13, 1969.

Speziale, C. G., Abid, R., and Anderson, E. C.: A critical evaluation of two-equation models for near wall turbulence, AIAA Paper 90-1481, 12, 1990.

Stillinger K. C., Helland, K. N., and van Atta, C. W.: Experiments on the transition of homogeneous turbulence to internal waves in a stratified fluid, J. Fluid Mech., 131, 91-122, 1983.

Tennekes, H.: The decay of turbulence in plane homogeneous shear flow, In Lecture Notes on Turbulence, edited by Herring, J. R. and McWilliams, J. C., World Scientific, 32-35, 1989.

Thompson, S. M. and Turner, J. S.: Mixing across an interface due to turbulence generated by an oscillating grid, J. Fluid Mech., 67, 349-368, 1975.

Townsend, A. A.: Equilibrium boundary layers and wall turbulence, J. Fluid Mech., 11, 97-120, 1961. 
Townsend, A. A.: The structure of turbulent shear flow, 2nd Ed., Cambridge University Press, 429, 1980.

Turner, J. S.: Small scale mixing processes, in: Evolution of Physical Oceanography, edited by Warren, B. A. and Wunsch, C., M.I.T. Press, 236-263, 1981.

Umlauf, L. and Burchard, H.: A generic length-scale equation for geophysical turbulence models, J. Mar. Sci., 61, 235-265 2003.

Umlauf, L., Burchard, H., and Hutter, K.: Extending the $k-\omega$ model towards oceanic applications, Ocean Modelling, 5, 195218, 2003.

Watmuff, J. H., Witt, H. T., and Joubert, P. N.: Developing turbulent boundary layers with system rotation, J. Fluid Mech., 157, 405-448, 1985.

Wigeland, R. A. and Nagib, H. M.: Grid-generated turbulence with and without rotation about the streamwise direction, Illinois Inst. of Tech. Fluids and Heat Transfer Rep. R78-1, 1978.

Wilcox, D. C.: A complete model of turbulence revisited, AIAA 22nd Aerospace Sciences Meeting, Reno, Nevada, January 9-12, 1984.

Wilcox, D. C.: Reassessment of the scale-determining equation for advanced turbulence models, AIAA J., 26, 1299-1310, 1988.

Wilcox, D. C.: Turbulence Modeling for CFD, 2. Ed., DCW Industries Inc, 1998.

Wilcox, D. C. and Traci, R. M.: A complete model of turbulence, AIAA Paper 76-351, July, 1976.

Wolfshtein, M., Naot, D., and Lin, A.: Models of turbulences, BenGurion University of Negev, Report ME-746, Israel, 1974.

Zeiermann, S. and Wolfshtein, M.: Turbulent time scale for turbulent-flow calculations, AIAA J., 24, 1606-1610, 1986. 